This is the peer reviewed version of the following article: Piri-Moghadam, H., Ahmadi, F., Gómez-Ríos, G. A., Boyacı, E., Reyes-Garcés, N., Aghakhani, A., . Pawliszyn, J. (2016). Fast Quantitation of Target Analytes in Small Volumes of Complex Samples by Matrix-Compatible Solid-Phase Microextraction Devices. Angewandte Chemie International Edition, 55(26), 7510-7514., which has been published in final form at https://doi.org/10.1002/anie.201601476. This article may be used for non-commercial purposes in accordance with Wiley Terms and Conditions for Self-Archiving.

\title{
Fast quantitation of target analytes in small volumes of complex samples by matrix-compatible Solid Phase Microextraction devices
}

\author{
Hamed Piri-Moghadam ${ }^{\dagger}$, Fardin Ahmadi ${ }^{\dagger}$, German Augusto Gómez-Ríos ${ }^{\dagger}$, Ezel Boyacl, Nathaly \\ Reyes-Garcés, Ali Aghakhani, Barbara Bojko and Janusz Pawliszyn*
}

\begin{abstract}
The current study reports the development of solid phase microextraction (SPME) devices designed to perform fast extraction/enrichment of target analytes present in small volumes of complex matrices (i.e. $V \leq 10 \mu \mathrm{L})$. Micro-sampling was performed with the use of etched metal tips coated with a thin layer of biocompatible nano-structured polypyrrole ( $P P_{Y}$ (tip diameter $\left.\leq 10 \mu \mathrm{m}\right)$, or by using coated blade spray (CBS) devices with an extraction phase thickness $\leq 10 \mu \mathrm{m}$, facilitating rapid extraction. These devices can be coupled either to liquid chromatography (LC), or directly to mass spectrometry (MS) via dedicated interfaces. The reported results demonstrated that the whole analytical procedure can be carried out within a few minutes (tanalysis $\leq 7 \mathrm{~min}$ ) with high sensitivity and quantitation precision, and can be used to sample from various biological matrices such as blood, plasma, and urine, towards the analysis of therapeutics, pesticides, and controlled substances as model analytes. As a proof-of-concept, the analysis and quantification of target compounds present in single cells of Allium cepa $L$ was demonstrated.
\end{abstract}

The development of new analytical technologies capable of providing high quantitation performance while delivering simplified and fast analysis of small amounts of biological samples (i.e. $\mathrm{V} \leq$ $10 \mu \mathrm{L})$ can undoubtedly impact the precision and efficiency of biological investigations in drug-
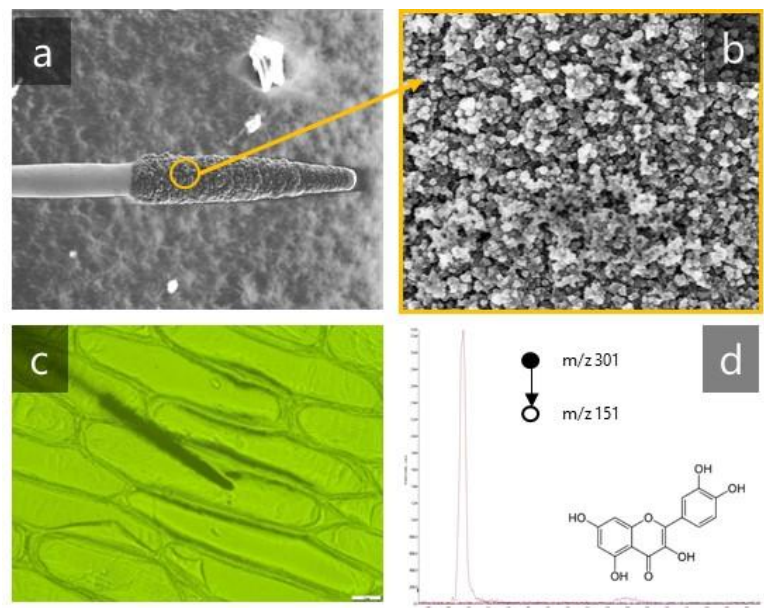

$8 \mathrm{~m} / \mathrm{z} 151$
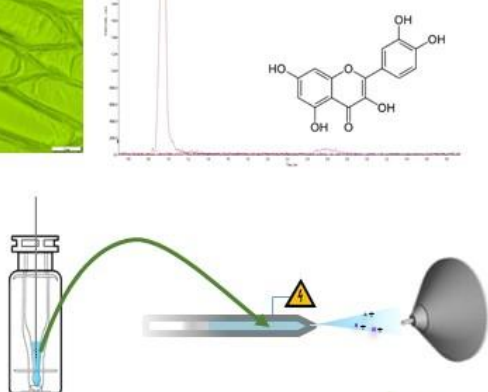

development and point-of-care (POC) diagnosis ${ }^{[1-}$ 4]. Facilitated by the improved sensitivity and specificity provided by modern mass spectrometry (MS) instrumentation, the replacement of traditional complex laboratory procedures with integrated miniaturized methods has become a growing trend in POC diagnosis ${ }^{[5]}$. In recent years, advances in direct sample to mass spectrometry techniques such as paper-spray ionization, probe electrospray ionization, and touch spray ${ }^{[2,5-7]}$ have allowed for the application of these methods towards the quantitative analysis of small volumes of biofluids. However, the sensitivity and precision typically achieved in the laboratory through adequate sample preparation prior to the MS quantification is traded off. Therefore, techniques capable of isolating and enriching target analytes from complex matrices with minimal processing time and adequate sample clean-up are highly desirable for applications that require direct introduction to MS ${ }^{[1-3,8,9]}$

As a concept, SPME embraces solventless microextraction technologies with different geometrical configurations that efficiently integrate sampling and sample clean-up, while also allowing for enrichment of the molar fraction of a given analyte in a single step ${ }^{[10]}$. Given the multiple advantages of this technique, including its feasibility to be coupled to different analytical instruments, SPME has been widely used for analysis of complex matrices such as biofluids, tissues, and food samples ${ }^{[1,9-12]}$.

The main objective of this report is to introduce two new SPME-based strategies that allow for the analysis of samples characterized by a small volume and/or size. Due to the thin nature of the coatings $(\varnothing \leq 10 \mu \mathrm{m})$, fast extraction/enrichment of target analytes can be achieved with the use of these devices, even when sampling from complex matrices. The proposed devices can be composed of either a mini-fibre (Figure 1), or a coated blade (Figure 2), allowing for extraction of analytes to be performed either by immersing the mini-fibre into the matrix, or by spotting the sample onto the coated blade. The miniaturized fibre is consisted of a polypyrrole ( $\mathrm{PPy}$ ) coated stainless steel micro-tip that can be conveniently interfaced with MS instrumentation via chromatography, or directly interfaced via nano-electrospray-ionization (nano-ESI). CBS, on the other hand, operates as a solidsubstrate electrospray ionization (ESI) source ${ }^{[8]}$. In the following paragraphs, the advantages and limitations of both approaches for the analysis of minute samples are concisely discussed. 
Figure 1 a. SEM image of a $150 \mu \mathrm{m}(100 x)$ tip coated with a 5 um laver of PPy-CTAB; $\boldsymbol{b}$. SEM image of nano-structured PPy-CTAB (5000x); c. $150 \mu \mathrm{m}$ tip inserted on a single cell of Allium cepa (red-onion); d. dhromatographic peak of quercetin extraded with mini-SPME from single calls of Allium cepa and identified by retention times and SRM $(301 \rightarrow 251 \mathrm{~m} / \mathrm{z})$ by ESI in negative mode; e. experimental set up when using mini-SPME directly coupled to MS via nano-ESI.

The use of miniaturized SPME probes not only facilitates the analysis of samples characterized by limited availability of sample (e.g. single cells $\left.{ }^{[13]}\right)$, but can also diminish potential damage caused during in vivo sampling ${ }^{[9]}$. Although the use of small metal probes for the analysis of single cells and tissue has already been reported ${ }^{[6,13]}$, due to the low sorption capacity and non-specific affinity of the metal surface (poor inter-device reproducibility), their application is limited to the determination of compounds present at high concentrations (e.g. phospholipids). In applications that require analysis of tissue or biofluids, device biocompatibility, defined not only as the capability to extract analytes of interest without significantly disturbing the system, but also in the sense of not coextracting cell substructures and macromolecules (e.g. organelles, proteins, lipoproteins) needs to be examined with extreme care ${ }^{[11,12]}$. If the chosen materials are insufficiently biocompatible with the

a

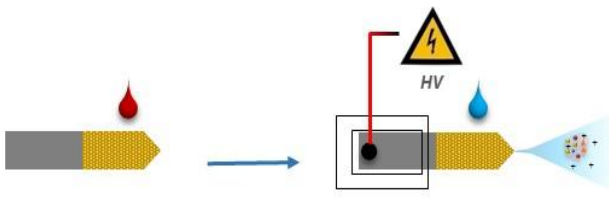

1. Extraction/sampling

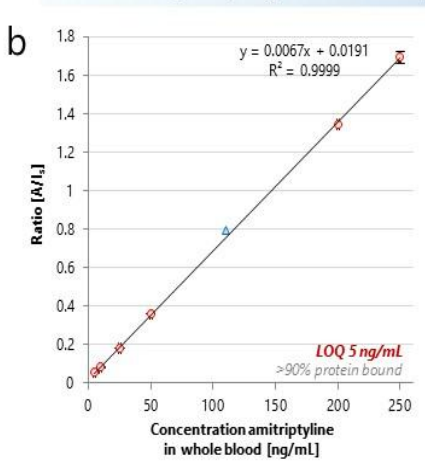

2. Rinsing

C Positive ionization

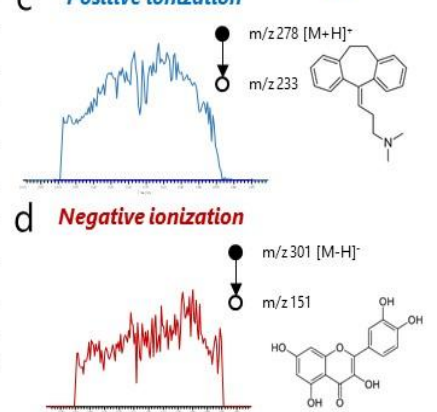

matrix under study, significant matrix effects are likely to occur, leading to poor or non-quantitation capabilities. ${ }^{[11]}$ Accordingly, the determination of compounds at trace levels in small volumes and single cells requires biocompatible polymeric coatings with high affinity toward the compounds of interest. PPy, a biocompatible $e^{[12]}$ material frequently used in biosensors ${ }^{[14]}$ and biomedical engineering ${ }^{[15]}$, is a well-known SPME extracting media for in vivo and in vitro bioanalysis ${ }^{[10]}$. However, the fabrication of miniature coated sampling devices has not been extensively explored to date due to the multiple challenges associated with the fabrication of such devices (e.g. mechanical stability and thickness of the coating). ${ }^{[10]}$ In this work, acupuncture needles with a diameter of $120 \mu \mathrm{m}$ were electrochemically etched to a tip with a diameter size of approximately $5 \mu \mathrm{m}$. Then, PPy was electrochemically coated onto the surface, using trifluoroacetic acid (TFA), and cetyltrimethylammonium bromide $(\mathrm{CTAB})^{[16]}$ as counter-ions (a detailed description of the experimental procedure is provided on the supporting information). Coatings of two different lengths, either of $150 \mu \mathrm{m}$ (conical-shape) or $500 \mu \mathrm{m}$ (cylindrical and conical shape), and with a coating thickness $\leq 5$ $\mu \mathrm{m}$ (see Figure S5), were applied onto the needles. Short coatings were intended to sample micro objects of interest such as drops, small organisms, or single cells, while cylindrical tips were envisioned to sample larger volumes with the aim to minimize invasiveness (e.g. in vivo sampling of brain tissue ${ }^{[9]}$ ). A comparison between the extraction capabilities of a PPy-CTAB coated tip and a bare wire showed that the extraction efficiency of the PPy-CTAB is at least 100 times higher than the one of the bare wire (Figures S9 and S10). In addition, as can be seen in Table S6, satisfactory results in terms of absolute matrix effects ${ }^{[11]}$ were found for analytes spiked on extracts of blood and plasma (i.e. 81-108 accuracy \%).

A remarkable feature of the tip configuration is its capability to perform extractions of target compounds in less than 2 min under static conditions (Figure S13). Certainly, the ability to perform rapid equilibration under static conditions can be beneficial for applications where agitation of the sample is not possible or cumbersome (e.g. analysis of tissue ${ }^{[10]}$, single cells ${ }^{[17]}$, and small samples $\left.{ }^{[4]}\right)$. The rapid rate of enrichment of analytes on the tip can be explained by radial diffusion (detailed discussion about radial diffusion and SPME devices in provided on the SI) ${ }^{[18]}$. Along with fast extraction times, the newly developed devices allow for fast desorption of analytes from the coating, an ideal feature that traditional SPME devices ${ }^{[10]}$ cannot easily achieve under static conditions ${ }^{[1]}$. As shown in Figure S14, by using a solvent with large affinity for the studied analytes, complete desorption of the extracted analytes can be achieved. This is particularly useful, for example, when performing direct coupling of SPME tips to MS via nano-ESI or through off-line desorption in few microliters of desorption solvent ${ }^{[1]}$, since in such cases, agitation cannot be applied to the fibre to accelerate the desorption of analytes.

Owing to the small size of the SPME tip, analysis of samples in the microliter range can be easily accomplished from a calibration perspective. Experimental findings showed non-statistical differences in terms of amount extracted when performing collection of analytes under equilibrium conditions on different volumes of whole blood sample (1-10 $\mu \mathrm{L}$, see Figure S15). The results obtained in PBS also showed that when samples larger than $5 \mu \mathrm{L}$ were used, negligible depletion could be assumed ${ }^{[12]}\left(K_{f s} V_{t i p}<<V_{s}\right)$ for most of the studied compounds, and that amounts extracted at equilibrium became independent of sample volume. As such, it can be concluded that there is minimum error associated with sampling of small volumes. Certainly, given the simplicity of the concentration calculations and the speed of analysis, SPME-tip is an ideal approach for analysis of small volumes of complex matrices. 
Figure 2 a. Experimental set up for analysis of -small volumes with coated blade spray. b. Quantitative analysis of whole blood spiked with amitriptyline (5 $\mathrm{ng} \mathrm{ml}^{-1}$ to $250 \mathrm{ng} \mathrm{mL}^{-1}$ ) and its isotopologue [D amitriptyline $\left(100 \mathrm{ng} \mathrm{mL}^{-1}\right) .5 \mu \mathrm{L}$ of sample were spotted on the blade, and 5 min static extraction/enrichment conducted using HLB-PAN coated blades. Bars represent the standard deviation of analyses for three replicates with independent blades. c. Ion dhronogram of amitriptyline; acquisition time of 30 $s$ in positive mode SRM $(278 \rightarrow 233 \mathrm{~m} / \mathrm{z})$; d. Ion dhronogram of quercetin; acquistion time of 30 s in negative mode; $\operatorname{SRM}(301 \rightarrow 251 \mathrm{~m} / \mathrm{z})$.

Quantitative determination of 5 test compounds (carbamazepine, propranolol, testosterone, diazepam, and pindolol) in $10 \mu \mathrm{L}$ of urine provided satisfactory figures of merit in the evaluated range (2-1000 ng mL $\mathrm{mL}^{-1}$; LOQ 3-10 $\mathrm{ng} \mathrm{mL}^{-1}$; see Table S8) and adequate intra-/inter-device reproducibility ( $\mathrm{RSD} \leq 20 \%$, see Table S9). As a proof of concept, microprobes were used for the qualitative analysis of Allium cepa (red-onion) single cells. Flavonoids, luteolin, and quercetin, previously reported on other food commodities, were successfully identified by comparing chromatographic retention times and fragmentation patterns with those of standards. As expected, target analytes could not be detected with the use of a non-coated tip; in contrast, good signal was achieved when the PPy-CTAB coated tip was exposed to the single cell (see Figure S19). However, application of the current device cannot prevent disturbances to the equilibrium of the investigated object, nor the cell damage that results from penetration of the membrane. Thus, smaller sampling means, such as a coated nano-tip ${ }^{[19]}$ with custom-made nanoparticles ${ }^{[20]}$, are under development, with aims to minimize these effects and make SPME suitable for cells one order of magnitude smaller (e.g. red-cells or fibroblasts). Aiming to have a better understanding of the quantitation power afforded by the PPyCTAB tips, direct coupling to MS instrumentation was performed via nano-ESI emitters (Figure 1) ${ }^{[1]}$. Quantitative determinations of therapeutics and drugs of abuse in urine, plasma, and blood were carried out by performing static extractions from $5 \mu \mathrm{L}$ of sample for 2 minutes. Subsequently, tips were rinsed for 3 seconds in water and then desorbed in $2 \mu \mathrm{L}$ of solvent for 2 minutes. Limits of quantitation (LOQs) in the low-ng/mL range were obtained for all target analytes, with a total analysis time of less than 5 minutes (Figure 3, S23, and S24).

Micro tips can effectively sample small volumes of selected parts of a living object. If the object is sufficiently large, the damage caused by insertion can be considered negligible or sufficiently minimized, allowing for multiple samplings to be subsequently performed to investigate changes in the system as a function of time or applied stimuli. However, if the sample is so small as to be substantially disturbed or consumed during extraction, or if the living object is so small as to not withstand sampling without loss of life, repetition of the sampling procedure is not possible. For such cases, exhaustive extraction on a coated larger flat surface might be a more attractive approach. To pursue this line of inquiry, CBS ${ }^{[8]}$ was implemented for the first time as a novel strategy for the analysis of small volumes of biofluids ${ }^{[8]}$. Unlike applications involving the tips, no additional instrumentation (i.e. LC or nanoESI emitter) or sample containers (e.g. vial) are needed to perform CBS analyses. As summarized in Figure 2, the analytical process consists of three simple steps: first, a drop of sample $(\leq 10 \mu \mathrm{L})$ is placed on the coated blade for 5 minutes (static conditions); then, the blade is rapidly rinsed in a vial containing water ( $t \leq 10 \mathrm{~s}$ ); and finally, the blade is installed in front of the MS system for analysis, as described by Gómez-Ríos et al ${ }^{[8]}$. As a sample preparation method, the coating (thickness $\leq 10 \mu \mathrm{m}$ ) simultaneously isolates and enriches the analytes present in the sample. Indeed, given the high affinity of $\mathrm{HLB}^{[11]}$ for a broad range of compounds, multiple rinsing steps can be performed to wash off matrix components potentially adhered to the surface with minimal to no loss of analytes. Thus, in comparison to directsample-to-mass spectrometry methods, CBS is capable of providing a significantly cleaner extract (i.e. minimal extraction of compounds that could cause ion-suppression or
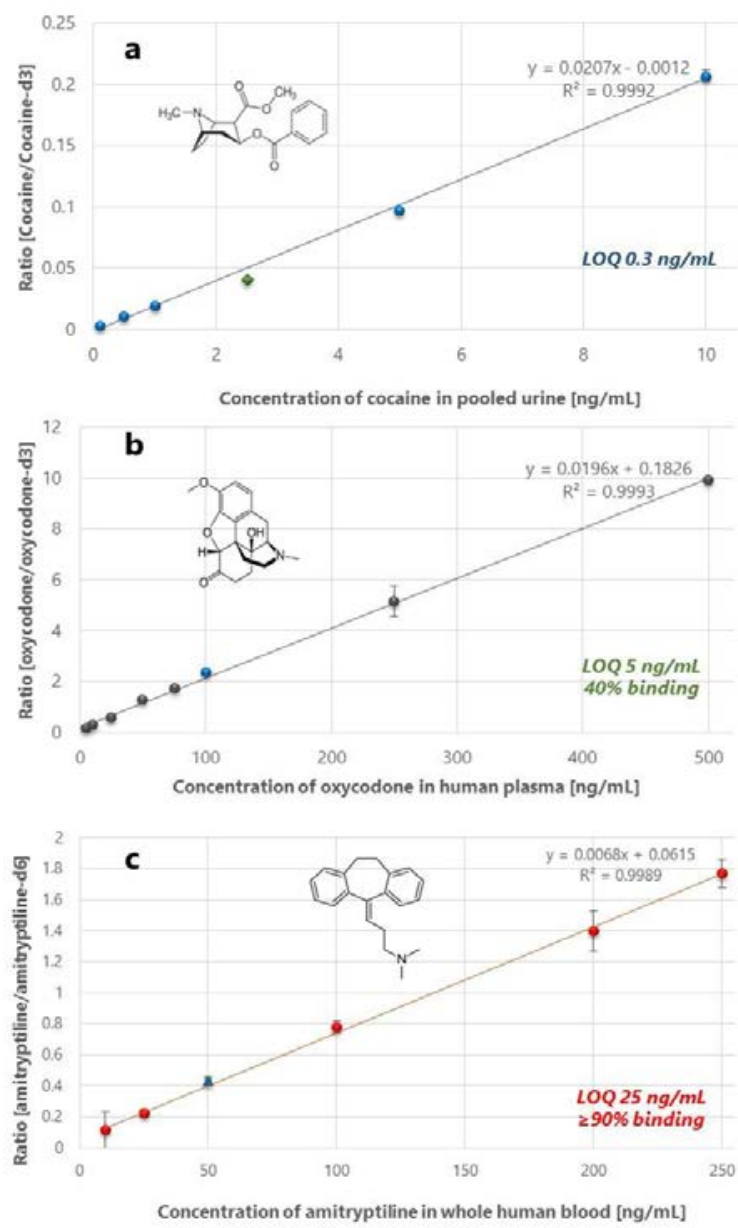

instrumental contamination). The use of cleaner extracts, in turn, allows for long-term instrument operation that requires minimal maintenance. Our results demonstrated that not only was good reproducibility attained (RSD $\leq$ $2 \%$, calculated based on the signal ratio of the analyte and its isotopologue $\left.\left[A / I_{s}\right]\right)$, but also noteworthy improvement of the LOQ for amitriptyline was achieved in comparison to that obtained with use of the tips. Essentially, the extraction efficiency of SPME techniques can be enhanced by increasing the affinity of the coating for the analytes (chemistry of the extraction phase) and/or by increasing the volume or active surface area of the extraction phase.

Figure 3 a. Quantitative analysis of urine spiked with cocaine $\left(0.1 \mathrm{ng} \mathrm{ml}^{-1}\right.$

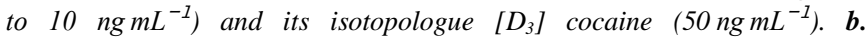
Quantitative analysis of plasma spiked with oxycodone $\left(1 \mathrm{ng} \mathrm{ml} \mathrm{m}^{-1}\right.$ to 500 $\left.n g \mathrm{~mL}^{-1}\right)$ and its isotopologue $\left[\mathrm{D}_{3}\right]$ oxycodone $\left(100 \mathrm{ng} \mathrm{mL}^{-1}\right)$. c. Quantitative analysis of whole blood spiked with amitriptyline $\left(5 \mathrm{ng} \mathrm{ml}^{-1}\right.$ to $\left.250 \mathrm{n} \mathrm{mL}^{-1}\right)$ and its isotopologue $\left[D_{6}\right]$ amitriptyline $\left(100 \mathrm{ng} \mathrm{mL}^{-1}\right) .5 \mu \mathrm{L}$ of 
sample was placed on a vial and analytes were extracted by a PPy-CTAB tip for 2 min under static conditions. All analyses were performed using nanoESI-MS/MS, with a total desorption volume of $2 \mu \mathrm{L}$ on MRM mode.

Certainly, the coated area of the blade where the droplet is placed significantly surpasses the total area of the SPME-tip. Therefore, in cases where both the affinity of the analyte for the coating and the extraction recovery are high (e.g. low matrixbinding), CBS should conceptually be considered as an open-bed SPE device, rather than as an SPME device. Finally, as a proofof-concept, CBS was used for the qualitative determination of quercetin in $5 \mu \mathrm{L}$ of homogenized onions (Figure 3). N egative ionization was used for identification of the analyte collected by the blade $(301 \rightarrow 251 \mathrm{~m} / \mathrm{z})$ after 5 minutes of contact. The obtained results evidence the great potential of CBS for fingerprinting and population studies of microorganisms such as cells, fungi, and bacteria. Hence, instead of inserting a mini-probe into a cell, single/multiple cells can be placed on top of the coated surface, and then burst to release their cytoplasmatic content. After allowing some time for extraction, the remaining cell components can then be washed, enabling subsequent determinations to be performed either by high-resolution (i.e. metabolite-profiling, Figure S26-27) or tandem mass spectrometry (i.e. target-analysis, Figure S28-31).

In conclusion, extraction rate enhancement can be achieved either by radial diffusion in the case of micro tips, or by high surface-to-volume ratio for blade format. Unlike other previously reported approaches that are based on metal needles without coating ${ }^{[6,7,19]}$, SPME acts as a chemical biopsy tool by enriching small molecules carrying chemical information about the investigated system without removing tissue, thus providing a much cleaner extraction. Currently, our group is working on the development of versatile biocompatible coatings for miniature devices that allow for the extraction of a broader range of compounds, with potential for a variety of applications in fields such as plant biology and oncology, where small objects need to be scrutinized. Likewise, the application of SPME tips for in-vivo tissue "imaging" is expected in the near future. Essentially, imaging should be understood as the capability of these miniature devices to monitor metabolite or drug concentration changes in specific regions of the tissue with minimum invasiveness (i.e. spatial resolution information). We foresee the SPME-tip device as a tool capable of complementing microdialysis and novel ambient ionization approaches suitable for the surgery room ${ }^{[21,22]}$. Certainly, our results also confirm CBS to be an effective enrichment and MS introduction tool, not only for point-of-care therapeutic drug monitoring, but also in diverse food, environmental, medical, and forensic applications where sample volume is limited, and in cases where a fast, disposable, deployable, sensitive, and selective quantitative tool is needed. Although the main document of this manuscript focuses on solvent-desorption applications, our initial endeavours towards the development of small SPME devices (i.e. Carbon Micro Fibres, CMF) suitable for thermal desorption applications such as gas chromatography (GC), direct-analysis in real time (DART), and other plasma-based sources are described on the supporting information material.

\section{Experimental Section}

SPME-tip experiments were performed with an API-4000 triple quadrupole mass spectrometer (SCIEX, Concord, ON, Canada) for quantitative analyses of target compounds by LC-MS/MS. SPME-
nano-ESI-MS/MS and CBS experiments were carried out on a TSQ Vantage and Orbitrap-Exactive mass spectrometers (Thermo Scientific, San Jose, CA, USA). Econo-10 and Glasstip emitters were obtained from New Objective (Woburn, MA, USA). CBS devices were manufactured according to an in-house coating procedure developed in our laboratory.

Received: ((will be filled in by the editorial staff))

Published online on ((will be filled in by the editorial staff))

Keywords: Biocompatible, miniature-SPME, single cell, sample preparation, mass spectrometry, coated blade spray, trace analysis, small volumes

[1] G. A. Gómez-Ríos, N. Reyes-Garcés, B. Bojko, J. Pawliszyn, Anal. Chem. 2016, 88, 1259-65.

[2] Y. Ren, M. N. McLuckey, J. Liu, Z. Ouyang, Angew. Chemie 2014, 126, 14348-14351.

[3] C. R. Ferreira, K. E. Yannell, A. K. Jarmusch, V. Pirro, Z. Ouyang, R. G. Cooks, Clin. Chem. 2015, 62, 99-110.

[4] A. Li, A. Hollerbach, Q. Luo, R. G. Cooks, Angew. Chem. Int. Ed. Engl. 2015, 54, 6893-5.

[5] D. T. Snyder, C. J. Pulliam, Z. Ouyang, R. G. Cooks, Anal. Chem. 2015, 88, 2-29.

[6] X. Gong, Y. Zhao, S. Cai, S. Fu, C. Yang, S. Zhang, X. Zhang, Anal. Chem. 2014, 86, 3809-16.

[7] K. Kerian, A. Jarmusch, R. Cooks, Analyst 2014, 2714-2720.

[8] G. A. Gómez-Ríos, J. Pawliszyn, Angew. Chemie 2014, 126, $14731-14735$

[9] E. Cudjoe, B. Bojko, I. de Lannoy, V. Saldivia, J. Pawliszyn, Angew. Chemie Int. Ed. 2013, 52, 12124-12126.

[10] É. A. Souza-Silva, N. Reyes-Garcés, G. A. Gómez-Ríos, E. Boyacı, B. Bojko, J. Pawliszyn, TrAC Trends Anal. Chem. 2015, $71,249-264$.

[11] N. Reyes-Garcés, B. Bojko, D. Hein, J. Pawliszyn, Anal. Chem. 2015, 87, 9722-9730.

[12] G. Ouyang, D. Vuckovic, J. Pawliszyn, Chem. Rev. 2011, 111, 2784-814.

[13] J. Deng, Y. Yang, M. Xu, X. Wang, L. Lin, Z.-P. Yao, T. Luan, Anal. Chem. 2015, 87, 9923-30.

[14] Y. Fang, Y. Ni, G. Zhang, C. Mao, X. Huang, J. Shen, Bioelectrochemistry 2012, 88, 1-7.

[15] X. Wang, X. Gu, C. Yuan, S. Chen, P. Zhang, T. Zhang, J. Yao, F. Chen, G. Chen, J. Biomed. Mater. Res. A 2004, 68, 411-22.

[16] S. Xing, G. Zhao, J. Appl. Polym. Sci. 2007, 104, 1987-1996.

[17] J. A. Stolee, B. Shrestha, G. Mengistu, A. Vertes, Angew. Chem. Int. Ed. Engl. 2012, 51, 10386-9.

[18] N. Godino, X. Borrise, F. X. Muñoz, F. J. Del Campo, R. G. Compton, J. Phys. Chem. C 2009, 113, 11119-11125.

[19] K. Zaitsu, Y. Hayashi, T. Murata, T. Ohara, K. Nakagiri, M. Kusano, H. Nakajima, T. Nakajima, T. Ishikawa, H. Tsuchihashi, et al., Anal. Chem. 2016, DOI 10.1021/acs.analchem.5b04046.

[20] T. Sun, Y. S. Zhang, B. Pang, D. C. Hyun, M. Yang, Y. Xia, Angew. Chem. Int. Ed. Engl. 2014, 53, 12320-64.

[21] J. Balog, S. Kumar, J. Alexander, O. Golf, J. Huang, T. Wiggins, N. Abbassi-Ghadi, A. Enyedi, S. Kacska, J. Kinross, et al., Angew. Chem. Int. Ed. Engl. 2015, DOI 10.1002/anie.201502770.

[22] A. K. Jarmusch, V. Pirro, Z. Baird, E. M. Hattab, A. A. CohenGadol, R. G. Cooks, Proc. Natl. Acad. Sci. U. S. A. 2016, 113, 1486-91. 
Entry for the Table of Contents (Please choose one layout)

Layout 1:

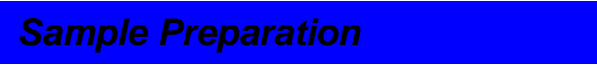

((Author(s), Corresponding Author(s)*)) Page - Page

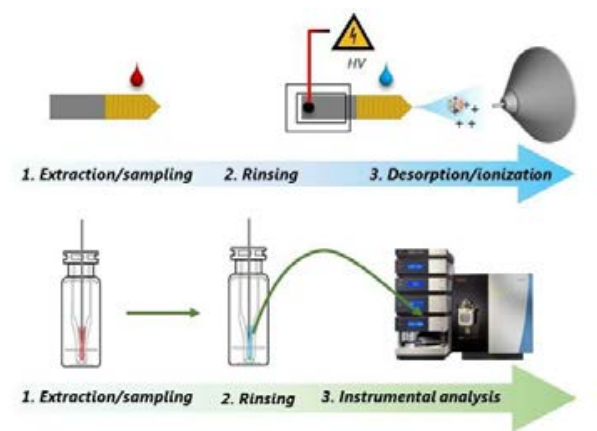

((Title Text))

Layout 2:

Page - Page

((Catch Phrase))
((Text for Table of Contents, max. 450 characters))

((TOC Graphic))

Page - Page 
